\title{
Estimación de la evapotranspiración mediante un balance de energía utilizando sensores remotos
}

\section{Estimation of evapotranspiration through an energy balance using remote sensors}

\author{
Carlos Raúl Castañeda-lbáñez ${ }^{1}$, Héctor Flores-Magdaleno ${ }^{1}$, Mario Martínez-Menes ${ }^{1}$, Salvador \\ Esparza-Govea ${ }^{1}$, Demetrio Fernández-Reynoso ${ }^{1}$, Víctor Prado-Hernández ${ }^{2}$, Fermín Pascual-Ramírez ${ }^{3 *}$ \\ ${ }^{1}$ Colegio de Postgraduados, Campus Montecillo, Carretera México-Texcoco km 36.5. CP. 56230. Texcoco, Estado de México, \\ México. \\ ${ }^{2}$ Universidad Autónoma Chapingo, Departamento de Suelos, Carretera México-Texcoco km 38.5. CP. 56230. Chapingo, \\ Texcoco, México. \\ ${ }^{3}$ CONACYT - Instituto de Investigaciones en Ecosistemas y Sustentabilidad. UNAM Campus Morelia. Antigua carretera a \\ Pátzcuaro \# 8701, Col. Exhacienda de San José de la Huerta. CP. 58190. Morelia, Michoacán de Ocampo, México. \\ *Autor de correspondencia: fpascualr@gmail.com
}

Artículo científico recibido: 09 de octubre de 2017 aceptado: 17 de mayo de 2018

RESUMEN. Determinar la evapotranspiración (ET) es importante en el proceso del uso y manejo del agua. Mapping Evapotranspiration at High Resolution using Internalized Calibration (METRIC) calcula la ET como un residual del balance de energía, por medio de imágenes de satélite. El objetivo fue validar el funcionamiento de METRIC para calcular la ET y estimar la eficiencia del uso del agua en el Distrito de Riego (DR) Rio Yaqui. Se utilizaron ocho imágenes de satélite Landsat (sensores TM y ETM+) y mediciones de flujos turbulentos con torres de covarianza turbulenta (Eddy Covariance - EC) en una parcela con cultivo de Trigo (Triticum aestivum) con extensión en todo el DR. La ET estimada con METRIC y la medida con EC mostraron alto grado de correspondencia, en las ocho imágenes se obtuvo una raíz cuadrada del cuadrado medio del error (RMSE) de $0.76 \mathrm{~mm} \mathrm{~d}^{-1}$, error medio absoluto (MAE) de $0.65 \mathrm{~mm} \mathrm{~d}^{-1}$, índice de acuerdo (d) de 0.93 y $\mathrm{R}^{2}$ de 0.83 en las estimaciones diarias. La metodología METRIC es una herramienta viable para estimar la variabilidad espacio-temporal de la ET y la eficiencia del uso del agua.

Palabras clave: Eficiencia, imágenes de satélite, METRIC, Triticum aestivum

ABSTRACT. Determining evapotranspiration (ET) is an important element in the water use and management process. Mapping Evapotranspiration at high Resolution with Internalized Calibration (METRIC) calculates ET as a residual of the energy balance using satellite images. The objective was to validate the functioning of METRIC to calculate the ET and estimate the efficiency of water use in the Rio Yaqui Irrigation District (ID). Eight Landsat satellite (TM and ETM+ sensors) images and turbulent flow measurements with turbulent covariance towers (Eddy Covariance EC) were used in a plot with common wheat (Triticum aestivum) cultivation extending throughout the DR. The ET estimated with METRIC and the measurement with EC showed a high degree of correspondence; in the eight images, a root-mean-square error (RMSE) of $0.76 \mathrm{~mm} \mathrm{~d}^{-1}$ was obtained, along with an absolute mean error (AME) of 0.65 $\mathrm{mm} \mathrm{d}^{-1}$, an agreement index (d) of 0.93 and an $\mathrm{R}^{2}$ of 0.83 in the daily estimates. The METRIC methodology is a viable tool to estimate the spatial, temporal variability of ET and the efficiency of water use.

Key words: Efficiency, evapotranspiration, satellite imagery, METRIC, Triticum aestivum

\section{INTRODUCCIÓN}

La evotranspiración (ET) es uno de los componentes más importantes del ciclo hidrológico, la mayor parte del agua que utilizan las plantas, la pierden por este fenómeno, por lo que los esfuerzos encaminados a mejorar la eficiencia en el uso del agua requieren de estimaciones confiables de la ET (Savage et al. 2009, Liu et al. 2012). La ET se estima con el producto de la evapotranspiración 
de referencia (ETr) y el coeficiente de cultivo $(\mathrm{Kc})$; para determinar el Kc se requiere conocer la etapa de desarrollo del cultivo, lo que representa una limitante en la estimación de la ET cuando se trabaja en áreas con diversidad y condiciones de desarrollo de cultivos (Allen et al. 2005a, 2005b). Aunque actualmente se cuenta con valores tabulados de Kc por cultivos y etapas de desarrollo, éstos fueron obtenidos para condiciones climáticas específicas en ambientes ideales de desarrollo (Neale et al. 1990). Estas limitaciones se incrementan cuando se pretende estimar la ET en áreas extensas, debido a la heterogeneidad en aspectos como el tipo de cultivo, fecha de siembra, condición de estrés y la etapa fenológica (Tasumi et al. 2005, Singh e Irmak 2009). Otros métodos para estimar la ET son el sistema del balance de energía de la relación de Bowen y las técnicas de Eddy Covariance (Ding et al. 2010), que consisten en medir los flujos de energía de la superficie del suelo a la atmósfera; los cuales se utilizan para hacer comparaciones con otras técnicas (Uddin et al. 2013, Bhattarai et al. 2017), validar estimaciones de modelos (Anderson et al. 2012, Liu et al. 2012) o para la determinación de Kc (Sun et al. 2010, Mateos et al. 2013, Saiz et al. 2017).

A pesar de que estos métodos permiten determinar con precisión la $\mathrm{ET}$, generalmente se realizan a nivel de parcela que resultan de utilidad limitada cuando se pretende cuantificar el consumo del agua a nivel regional, debido al costo y el tiempo que representa hacer suficientes mediciones para realizar estimaciones precisas de la ET (Irmak et al. 2011). Los modelos de balance de energía superficial acoplados a información obtenida por sensores a bordo de plataformas satelitales se han aplicado para la estimación de la ET (Allen et al. 2007a, Singh et al. 2017); en tanto que, en décadas recientes, las técnicas de percepción remota, se han utilizado con éxito en la gestión del agua de riego (Calera et al. 2005, Palacios-Vélez et al. 2018).

METRIC es un algoritmo que estima la ET como un residual del balance de energía utilizando imágenes de satélite (Allen et al. 2007b) a partir de la concepción y desarrollo del SEBAL (Sur- face Energy Balance Algorithms for Land), que fue diseñado para cuantificar ET sobre extensas áreas, estimando los flujos del balance de energía mediante el uso de sensores remotos (Bastiaanssen 1998). Para su funcionamiento, METRIC, utiliza información en el espectro visible, infrarrojo cercano y en la banda térmica; que se ha utilizado en diferentes lugares en la estimación de ET a escala diaria, mensual o de estación completa (Santos et al. 2010, Senay et al. 2011, French et al. 2015). Las principales aplicaciones son en la planeación del manejo y derechos administrativos del agua (Irmak et al. 2011, Hankerson et al. 2012, Mkhwanazi et al. 2012). También se ha utilizado en la estimación de la tasa de evaporación en cuerpos de agua con buen desempeño en sus resultados (Zamani y Rahimzadegan 2018). Por lo anterior, el objetivo del presente estudio fue validar el funcionamiento de METRIC en la estimación de la distribución espacial y temporal de la ET, para conocer la eficiencia en el uso del agua durante el ciclo del cultivo de trigo en el Distrito de Riego del Rio Yaqui, Sonora.

\section{MATERIALES Y MÉTODOS}

El Distrito de Riego 041 Río Yaqui, también denominado Valle del Yaqui tiene una superficie de 223000 ha, se ubica al Noroeste de México, abarcando los municipios de Cajeme, Bacum, San Ignacio Rio Muerto, Benito Juárez, Etchojoa y Navojoa en el estado de Sonora, entre las coordenadas extremas $26^{\circ} 45^{\prime}$ y $27^{\circ} 40^{\prime} \mathrm{LN}$ y $109^{\circ} 45^{\prime}$ y $110^{\circ} 20^{\prime}$ LO.

Se estimó la ET con el método METRIC con ocho imágenes de los sensores TM y ETM+ de los satélites LANDSAT 5 y 7 (Path: 34, Row: 41), que se obtuvieron del Servicio Geológico de los Estados Unidos (USGS). Se seleccionaron las imágenes del 15 de enero, ocho y 24 de febrero, 11 y 27 de marzo, 12 y 28 de abril, y 14 de mayo de 2008; las fechas se convirtieron a días juliano del 2008, resultando los días 15, 39, 55, 71, 87, 103, 119 y 135. Para calcular el balance de energía, se utilizó la aplicación Macro Modeler del Software ERDAS Imagine System; también se utilizó el Software ArcGis 10 para la 
visualización de imágenes. La validación de la precisión y el funcionamiento del algoritmo se realizó con mediciones de flujos turbulentos con torres de Eddy Covariance (EC) (Palacios-Vélez et al. 2011), tomando como referencia una parcela de trigo con ciclo de producción del 31 de diciembre de 2007 al 14 de mayo de 2008.

Se utilizó el método Cubic-Spline para la interpolación de la ET (Singh et al. 2012) en la obtención del mapa de la ET total por mes (METRIC), con lo que se obtuvo la ET del ciclo de desarrollo del trigo. El mensual evapotranspirado (EC) se obtuvo de la suma de los valores diarios reportados por la torre Eddy Covariance en cada uno de los meses analizados. Para evaluar el funcionamiento del algoritmo a nivel diario, mensual y del ciclo de desarrollo del cultivo, se identificó la parcela en la que se realizaron mediciones de evapotranspiración con EC y se compararon los valores estimados con METRIC en la fecha de obtención de cada imagen. Para la validación, se analizó el error medio absoluto (MAE) y la raíz cuadrada del cuadrado medio del error (RMSE) como medidas de precisión, mientras que de variabilidad se analizó el coeficiente de correlación $\left(\mathrm{R}^{2}\right)$ y el índice de acuerdo $(\mathrm{d})$, para conocer la relación que existe entre la ET medida y la predicha por el modelo.

\section{Estimación de la eficiencia del uso del agua en el distrito}

Para calcular la eficiencia del uso del agua se analizó de forma conjunta la ET (METRIC) con los volúmenes de agua consumidos (Tabla 1). El volumen aportado por la precipitación efectiva se estimó con el método USDA en los meses de diciembre a mayo. En el estado de Sonora, la mayor precipitación $(\mathrm{P})$ y la precipitación efectiva $(\mathrm{Pe})$ se presenta de julio a septiembre y, en menor medida, de octubre a enero donde la presencia de frentes fríos favorece la ocurrencia de lluvia.

\section{RESULTADOS}

\section{Validación del algoritmo METRIC con estima-} ciones diarias

Los valores estimados de ET diaria obtenidos mediante METRIC y los reportados por la torre de Eddy Covariance presentan contrastes en los valores obtenidos (Tabla 2). Con ambos métodos y diferentes fechas de las imágenes, los valores de ET diaria varían en cerca de $1 \mathrm{~mm}$ para el primer mes e incrementa hasta valores de alrededor de $6 \mathrm{~mm} \mathrm{~d}^{-1}$; con METRIC, este valor se alcanza en abril, en tanto que con el método de Eddy Covariance se encuentra a mediados de marzo. Ambos métodos estiman la ET de $3 \mathrm{~mm}$ en el último mes de desarrollo del cultivo. Las imágenes procesadas con METRIC de los días julianos 15, 39, 71 y 135 , subestiman el valor de la ET con $0.4,0.1,0.6$ y $0.2 \mathrm{~mm}$, respectivamente. Mientras que los días julianos 55, 87, 103 y 119, tienen un valor mayor del medido con EC de 0.9, $0.6,1.1$ y $1.4 \mathrm{~mm}$, respectivamente. Las diferencias más significativas las presentan las imágenes de los días julianos 15, 103 y 119 con el 28.6, 22.4 y el $36.8 \%$ de error, respectivamente; al inicio y final de la estación de crecimiento del cultivo.

\section{Validación del algoritmo METRIC con estima- ciones mensuales}

Para las estimaciones mensuales, los errores se reducen con relación a las estimaciones diarias. El error reportado en el mes de enero es el más alto y se debe a los bajos valores del Kc para esas fechas, ya que es el inicio de la estación de crecimiento en las parcelas de trigo, y las condiciones son diferentes a las de los pixeles de referencia que están completamente desarrollados, cubiertos por vegetación y que evapotranspiran más del $100 \%$ de la ETr (Tabla 3).

Validación del algoritmo METRIC para la
estación completa de desarrollo del trigo
El análisis de las imágenes mensuales generadas con METRIC permitió sumar los valores de ET mensuales y obtener un mapa de la ET total del 31 de diciembre del 2007 al 14 de mayo del 2008 
Tabla 1. Volumen de agua utilizada y precipitación mensuales en el distrito de riego.

\begin{tabular}{ccccc}
\hline \multicolumn{2}{c}{ Volumen a nivel de presa y pozos profundos } & \multicolumn{2}{c}{ Precipitación } \\
\hline Año & Mes & Miles de $\mathrm{m}^{3}$ & $\mathrm{P}(\mathrm{mm})$ & $\mathrm{Pe}(\mathrm{mm})$ \\
\hline 2007 & Diciembre & 81406.22 & 51.4 & 47.2 \\
2008 & Enero & 145267.12 & 4.3 & 4.2 \\
2008 & Febrero & 346937.97 & 0.7 & 0.69 \\
2008 & Marzo & 415488.86 & 10.2 & 10 \\
2008 & Abril & 234936.92 & 0 & 0 \\
2008 & Mayo & 46241.77 & 0 & 0 \\
\hline
\end{tabular}

Tabla 2. Estimaciones diarias de ET

\begin{tabular}{ccccccc}
\hline \multirow{2}{*}{ Fecha de imagen } & \multicolumn{1}{c}{ Día del Año } & METRIC & Eddy Covariance & \multirow{2}{*}{ Diferencia } & \% Error \\
\cline { 2 - 3 } & \multicolumn{3}{c}{ Evapotranspiración $\left(\mathrm{mm} \mathrm{d}^{-1}\right)$} & & \\
\hline $15 / 01 / 2008$ & 15 & 1.0 & 1.4 & 0.4 & 28.6 \\
$08 / 02 / 2008$ & 39 & 3.4 & 3.5 & 0.1 & 2.9 \\
$24 / 02 / 2008$ & 55 & 5.1 & 4.2 & -0.9 & -21.4 \\
$11 / 03 / 2008$ & 71 & 5.6 & 6.2 & 0.6 & 9.7 \\
$27 / 03 / 2008$ & 87 & 5.7 & 5.1 & -0.6 & -11.8 \\
$12 / 04 / 2008$ & 103 & 6.0 & 4.9 & -1.1 & -22.4 \\
$28 / 04 / 2008$ & 119 & 5.2 & 3.8 & -1.4 & -36.8 \\
$14 / 05 / 2008$ & 135 & 3.0 & 3.2 & 0.2 & 6.2 \\
\hline
\end{tabular}

Tabla 3. Estimaciones mensuales de ET.

\begin{tabular}{|c|c|c|c|c|}
\hline \multirow[t]{2}{*}{ Mes } & METRIC & Eddy Covariance & \multirow[t]{2}{*}{ Diferencia } & \multirow[t]{2}{*}{ Error \% } \\
\hline & \multicolumn{2}{|c|}{ Evapotranspiración $\left(\mathrm{mm} \mathrm{d}^{-1}\right)$} & & \\
\hline Enero & 42.0 & 67.3 & 25.3 & 37.6 \\
\hline Febrero & 91.8 & 89.3 & -2.5 & -2.8 \\
\hline Marzo & 168.3 & 155.0 & -13.3 & -8.6 \\
\hline Abril & 158.3 & 131.2 & -27.1 & -20.7 \\
\hline Mayo & 54.2 & 49.7 & -4.5 & -9.0 \\
\hline
\end{tabular}

para los diferentes cultivos establecidos en Valle del Yaqui (Figura 1). En los primeros días del año, los valores bajos de ET, identificados con colores obscuros, se deben a la presencia de parcelas de riego con poca vegetación que corresponden al ciclo de cultivo anterior y, al iniciar el lapso evaluado, están al final de su etapa de desarrollo, por lo cual en la mayoría de los casos se asume que son pixeles de parcelas secas con ET cercana a cero.

\section{Eficiencia del uso del agua en el distrito 041}

Más del $86 \%$ de la superficie del distrito está ocupada por el trigo en el ciclo de cultivo otoñoinvierno, que se siembra desde mediados de noviembre hasta mediados de diciembre, y las fechas límites para la cosecha están establecidas para el 30 de mayo. Para la ET calculada, se conoce el volumen de agua utilizado para el riego de cultivos establecidos dentro del Distrito, por lo que se pueden hacer estimaciones de la eficiencia del riego para la duración del ciclo del cultivo.

El volumen evapotranspirado en el distrito, estimado mediante METRIC para el ciclo del cultivo fue de $938251.91 \mathrm{Mm}^{3}$, mientras que el volumen de agua aplicado fue de $1270278.86 \mathrm{Mm}^{3}$, y el volumen aportado por la precipitación efectiva fue de $137038.08 \mathrm{Mm}^{3}$. Lo que indica una eficiencia del uso del agua del $66 \%$.

\section{DISCUSIÓN}

Las diferencias en la ET estimada con METRIC y la estimada con EC muestran un alto grado de aceptación, para las 8 imágenes se obtuvo un RMSE de $0.76 \mathrm{~mm}$ y un error medio absoluto (MAE) de $0.65 \mathrm{~mm}$. El índice de acuerdo (d) es de 0.93, mientras que los valores de ET estimados con METRIC y EC se ajustan a un modelo 


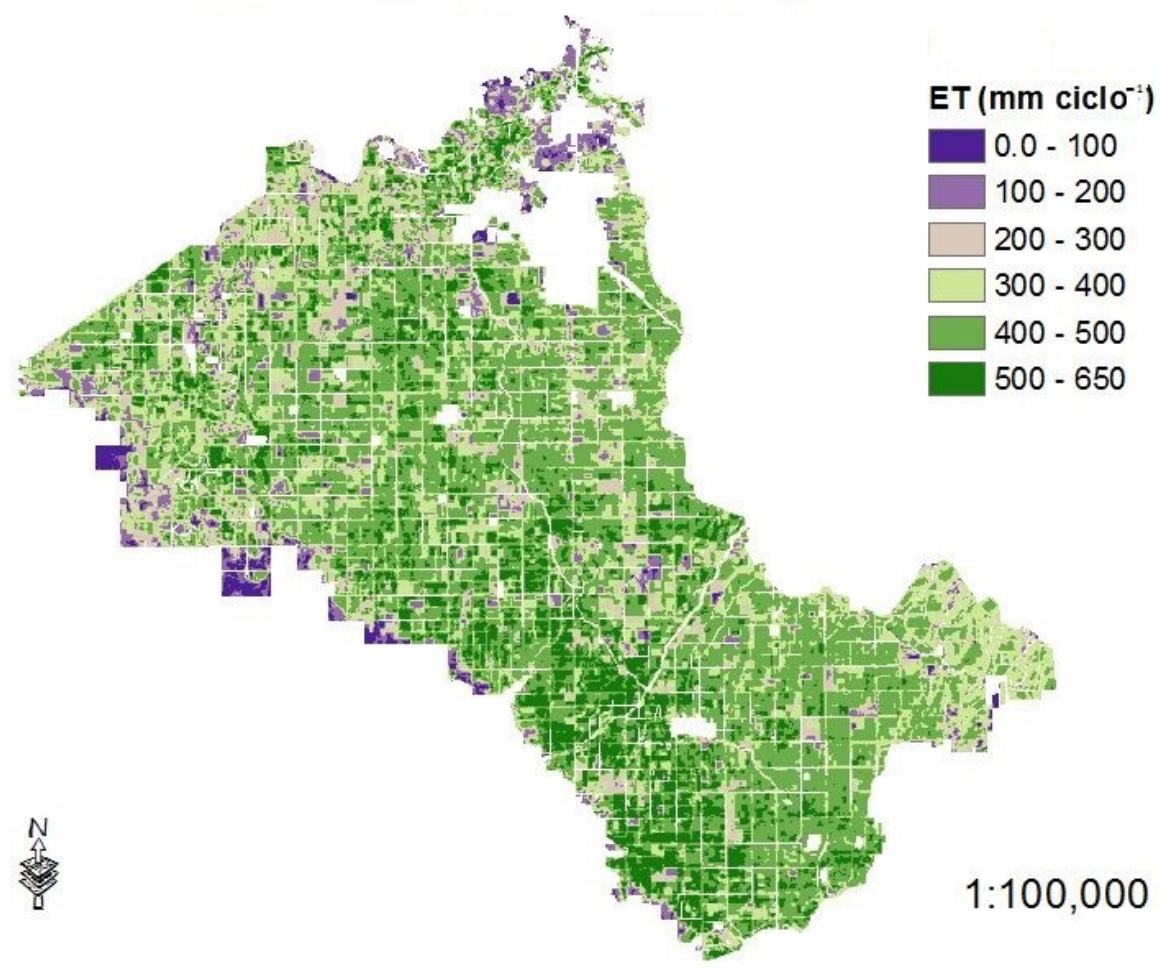

Figura 1. Distribución de la evapotranspiración total del ciclo.

lineal con coeficiente de determinación de $\mathrm{R}^{2}=$ 0.83 y correlación de 0.90 (Figura 2). Por otra parte, si en la regresión se asume intersección de cero $\left(R^{2}=0.79\right)$ METRIC subestimará a nivel diario aproximadamente el $10 \%$, lo que es aceptable cuando se están realizando estimaciones de bajo costo en grandes superficies agrícolas. Resultados similares fueron reportados por Pôças et al. (2014) al ampliar la temporalidad de aplicación del modelo en conjunto con información meteorológica de campo.

En los meses de rápido crecimiento (marzo y abril) METRIC sobre estimó los valores de ET a pesar de reportes que indican mejor desempeño en cultivos de alta densidad y bajo porte (Bhattarai et al. 2017). En estas condiciones, para aumentar la precisión, es importante el estricto control de calidad de los datos meteorológicos al estimar los valores diarios de ET. Debido a que las diferencias (EC y METRIC) son en milímetros por mes, se consideran poco significativos $\left(\mathrm{MAE}=9.08 \mathrm{~mm} \mathrm{mes}{ }^{-1}, \mathrm{RMSE}\right.$ $=17.76 \mathrm{~mm}, \mathrm{~d}=0.99$ ), por lo cual el grado de aceptación de las estimaciones mensuales es alto, incluso mayor que las estimaciones diarias; resultados que son más precisos que los reportados con SEBAL en condiciones similares (Singh et al. 2017). Los valores de ET mensuales interpolados y calculados con EC se ajustaron a un modelo de regresión lineal con $\mathrm{R}^{2}$ de 0.93 y coeficiente de correlación de 0.96 (Figura 3). Si en el modelo de regresión lineal se asume intersección en cero, el coeficiente de determinación sigue siendo aceptable $\left(R^{2}=0.86\right)$. De acuerdo con los indicadores analizados $\left(R^{2}, M A E\right.$, RMSE, d) a pesar de los sesgos de las estimaciones diarias, las interpolaciones mensuales reducen los errores y le dan a METRIC mayor confiabilidad en las estimaciones de ET, aunque es importante indicar que la precisión varía en función del periodo de análisis y de la representatividad de los pixeles extremos seleccionados (Senay et al. 2011). 


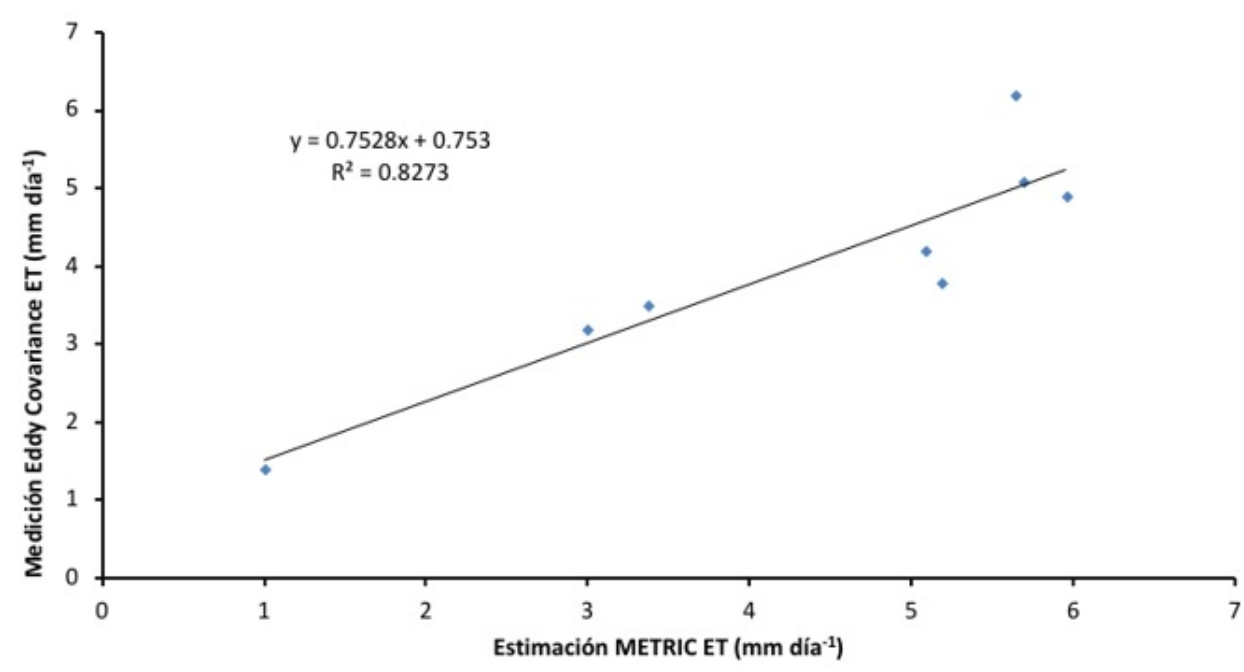

Figura 2. Modelo de regresión lineal de la ET estimada y medida diaria.

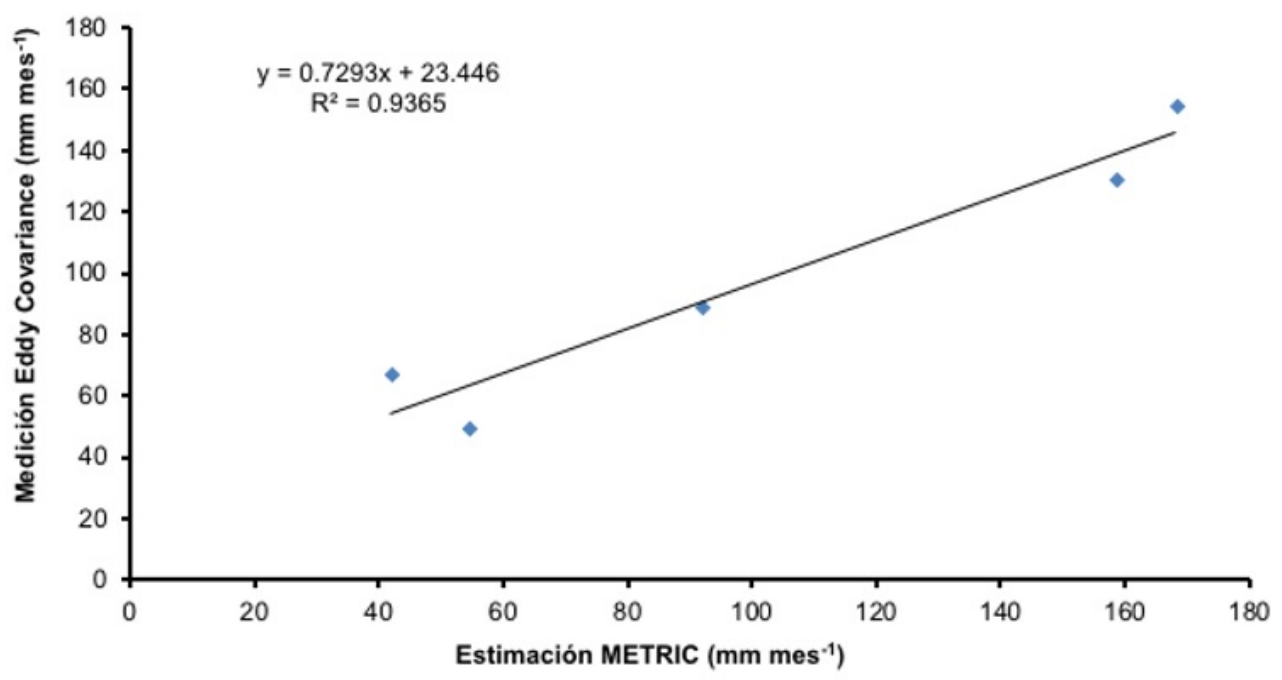

Figura 3. Relación mensual de la ET estimada y medida.

En la parcela bajo estudio, considerando el ciclo completo del cultivo, la ET estimada con METRIC (514.6 $\mathrm{mm}$ ) resultó mayor que la medida con EC (492.5 mm) por apenas el $4.4 \%$, en general, son similares a los valores encontrados utilizando SEBAL al considerar el ciclo completo del cultivo con la misma estación de cultivo (Jian-Ying et al. 2015). Los resultados son acordes a la lámina evapotranspirada de $429 \mathrm{~mm}$ encontrada en un estudio del uso consuntivo del agua por el cultivo de trigo en el Valle del Yaqui con métodos tradicionales (De la Peña y Llerena 2001). Al analizar la totalidad del área de interés, se encontró que el volumen promedio evapotranspirado fue de $400 \mathrm{~mm}$ y cerca del $70 \%$ de la superficie evapotranspiró entre 300 y $500 \mathrm{~mm}$ (Tabla 4).

Los resultados de la validación del METRIC son similares a los reportadas por Irmak et al. (2011) quienes reportan coeficientes de correlación de 0.88. Al respecto Mkhwanazi et al. (2012) re- 
Tabla 4. Rangos de ET por superficie en el ciclo de desarrollo del trigo.

\begin{tabular}{ccc}
\hline Rango ET $\left(\mathrm{mm}\right.$ ciclo $\left.^{-1}\right)$ & Superficie $($ ha $)$ & Total $(\%)$ \\
\hline $0.0-100$ & 3114.63 & 1.36 \\
$100-200$ & 11943.81 & 5.23 \\
$200-300$ & 23956.83 & 10.48 \\
$300-400$ & 55315.71 & 24.21 \\
$400-500$ & 105012.90 & 45.96 \\
$500-650$ & 29156.22 & 12.76 \\
\hline
\end{tabular}

portan coeficientes de regresión de 0.98 con errores en las estimaciones del $5 \%$, mientras que Allen et al. (2007a) reportan errores promedio en diferentes cultivos del $4 \%$, lo que es similar al error del $4.4 \%$ encontrado. Comparado con otros métodos que utilizan información de imágenes satelitales, METRIC tiene mayor confiabilidad cuando los datos terrestres son escasos (French et al. 2015), además de mejor sensibilidad en diferentes etapas de desarrollo de los cultivos. Cuando el trigo se encuentra en etapa de desarrollo (febrero, marzo, mediados de abril), hay mínimas diferencias significativas en la estimación de la ET (Hankerson et al. 2012, Singh et al. 2017), pero incrementan cuando el cultivo está al inicio o final de su ciclo fenológico (Tabla 3). Por otro lado, los mayores errores se presentan en cálculos diarios que al integrarlos a menor resolución temporal (mensual, estacional) disminuyen, mejorando la precisión. Otros estudios, reportan que METRIC puede mejorar la estimación de la ET, si el proceso de ejecución se hace diferenciando la temperatura del dosel, del suelo sombreado y del suelo a libre iluminación solar (Pôças et al. 2014). Con respecto a la estimación de la eficiencia en el uso del agua, la estimación puede mejorar si se conoce el gasto aplicado a mayor resolución espacial y temporal para detectar las áreas con menor eficiencia en el uso del agua en el distrito de riego. Por lo que los resultados adolecen de la validación que permita contribuir a mejorar el uso y manejo del agua al proponer acciones para incrementar la eficiencia.

\section{CONCLUSIONES}

METRIC es una alternativa viable para estimar la ET en regiones agrícolas, la validación del algoritmo presenta errores promedio menores a un milímetro para estimaciones diarias. El análisis del ciclo de crecimiento del cultivo, mostró que los errores son compensados y se reducen a valores de alrededor del $4.4 \%$. Los resultados sugieren que METRIC se puede aplicar a series de tiempo de imágenes de satélite en estudios sobre la distribución espacial y temporal de la evapotranspiración que conduzcan a implementar acciones para mejorar el uso del agua en extensas áreas agrícolas de riego.

\section{AGRADECIMIENTOS}

Al Proyecto CONACYT PDCPN 2015-640 'Bases para mejorar el aprovechamiento sustentable del agua de uso agrícola en México' y al Proyecto Cátedras CONACYT 2269. Al CONACYT, por el financiamiento de los estudios de maestría del primer autor.

\section{LITERATURA CITADA}

Allen RG, Pereira LS, Smith M, Raes D, Wright JL (2005a) FAO-56 dual crop coefficient method for estimting evaporation from soil and application extensions. Journal of Irrigation and Drainage Engineering 131: 2-13.

Allen RG, Tasumi M, Morse A, Trezza R (2005b) A Landsat-based energy balance and evapotranspiration model in Western US water rights regulation and planning. Irrigation and Drainage Systems 19: 251268. 
Allen RG, Tasumi M, Morse A, Trezza R, Wright JL, Bastiaanssen W, et al. (2007a) Satellite-based energy balance for mapping evapotranspiration with internalized calibration (METRIC) -applications. Journal of Irrigation and Drainage Engineering 133: 395-406.

Allen RG, Tasumi M, Trezza R (2007b) Satellite-based energy balance for mapping evapotranspiration with internalized calibration (METRIC)-Model. Journal of Irrigation and Drainage Engineering 133: 380-394.

Anderson RG, Jin Y, Goulden ML (2012) Assessing regional evapotranspiration and water balance across a Mediterranean montane climate gradient. Agricultural and Forest Meteorology 166-167: 10-22.

Bastiaanssen W, Menenti M, Feddes R, Holtslag A (1998) A remote sensing surface energy balance algorithm for land (SEBAL). 1. Formulation. Journal of Hydrology 212: 198-212

Bhattarai N, Quackenbush L, Im J, Shaw S (2017) A new optimized algorithm for automating endmember pixel selection in the SEBAL and METRIC models. Remote Sensing of Environment 196: 178-192.

Calera A, Jochum AM, García AC, Rodríguez AM, Fuster PL (2005) Irrigation management from space: Towards user-friendly products. Irrigation and Drainage Systems 19: 337-353.

De la Peña I, Llerena F (2001) Manual del uso y manejo del agua de riego, 3rd ed. Editorial Futura. México. $216 \mathrm{p}$.

Ding R, Kang S, Li F, Zhang Y, Tong L, Sun Q (2010) Evaluating eddy covariance method by large-scale weighing lysimeter in a maize field of northwest China. Agricultural Water Management 98: 87-95.

French AN, Hunsaker DJ, Thorp KR (2015) Remote sensing of evapotranspiration over cotton using the TSEB and METRIC energy balance models. Remote Sensing of Environment 158: 281-294.

Hankerson B, Kjaersgaard J, Hay C (2012) Estimation of evapotranspiration from fields with and without cover crops using remote sensing and in situ methods. Remote Sensing 4: 3796-3812.

Irmak A, Ratcliffe I, Ranade P, Hubbard KG, Singh RK, Kamble B, et al. (2011) Estimation of land surface evapotranspiration with a satellite remote sensing procedure. Great Plains Research 21: 73-88.

Jian-ying Y, Xu-rong M, Chang-rong Y, Hui J, Feng-hua Z, Qin L (2015) Water consumption in summer maize and winter wheat cropping system based on SEBAL model in Huang-Huai-Hai Plain, China. Journal of Integrative Agriculture 14: 2065-2076

Liu G, Liu Y, Hafeez M, Xu D, Vote C (2012) Comparison of two methods to derive time series of actual evapotranspiration using eddy covariance measurements in the southeastern Australia. Journal of Hydrology 454-455: 1-6.

Mateos L, González-Dugo MP, Testi L, Villalobos FJ (2013) Monitoring evapotranspiration of irrigated crops using crop coefficients derived from time series of satellite images. I. Method validation. Agricultural Water Management 125: 81-91.

Mkhwanazi M, Chávez J, Rambikur E (2012) Comparison of large aperture scentillometer and satellite-based energy balance models in sensible heat flux and crop evapotranspiration determination. International Journal of Remote Sensing Applications 2: 24-30.

Neale C, Bausch W, Heermann D (1990) Development of Reflectance-Based Crop Coefficients for Corn. Transactions of the ASAE 32: 1891-1900.

Palacios-Vélez E, Palacios-Sánchez J, Palacios L (2011) Agricultura de riego asistida con satélites. Tecnología y Ciencias del Agua 2: 69-81. 
Palacios-Vélez E, Palacios-Sánchez L, Espinosa-Espinosa J (2018) Evaluation of water use efficiency in irrigation agriculture supported by satellite images. Tecnología y Ciencias del Agua 9: 31-38.

Pôças I, Paço TA, Cunha M, Andrade JA, Silvestre J, Sousa A, et al. (2014) Satellite-based evapotranspiration of a super-intensive olive orchard: Application of METRIC algorithms. Biosystems Engineering 128: $69-81$.

Saiz R, Aguirre G, Rodriguez J, Watts C, Saiz J, Ochoa A, Saiz J (2017) Estimación de evapotranspiración con imágenes de PROBA-V de un cultivo de sandía en la costa de Hermosillo, Sonora, México. Terra Latinoamericana 35: 301-308.

Santos C, Lorite IJ, Tasumi M, Allen RG, Fereres E (2010) Performance assessment of an irrigation scheme using indicators determined with remote sensing techniques. Irrigation Science 28: 461-477.

Savage MJ, Everson CS, Metelerkamp BR (2009) Bowen ratio evaporation measurement in a remote montane grassland: Data integrity and fluxes. Journal of Hydrology 376: 249-260.

Senay GB, Budde ME, Verdin JP (2011) Enhancing the simplified surface energy balance (SSEB) approach for estimating landscape ET: Validation with the METRIC model. Agricultural Water Management 98: 606-618.

Singh RK, Irmak A (2009) Estimation of crop coefficients using satellite remote sensing. Journal of Irrigation and Drainage Engineering 135: 597-608.

Singh RK, Liu S, Tieszen LL, Suyker AE, Verma SB (2012) Estimating seasonal evapotranspiration from temporal satellite images. Irrigation Science 30: 303-313.

Singh RK, Bala A, Kumar S, Kumar R (2017) Quantification of wheat crop evapotranspiration and mapping: A case study from Bhiwani District of Haryana, India. Agricultural Water Management 187: 200-2009.

Sun G, Noormets A, Gavazzi MJ, McNulty SG, Chen J, Domec JC, et al. (2010) Energy and water balance of two contrasting loblolly pine plantations on the lower coastal plain of North Carolina, USA. Forest Ecology and Management 259: 1299-1310.

Tasumi M, Allen RG, Trezza R, Wright JL (2005) Satellite-based energy balance to assess within-population variance of crop coefficient curves. Journal of Irrigation and Drainage Engineering 131: 94-109.

Uddin J, Hancock NH, Smith RJ, Foley JP (2013) Measurement of evapotranspiration during sprinkler irrigation using a precision energy budget (Bowen ratio, eddy covariance) methodology. Agricultural Water Management 116: 89-100.

Zamani S, Rahimzadegan M (2018) Evaluation of SEBS, SEBAL, and METRIC models in estimation of the evaporation from the freshwater lakes (Case estudy: Amirkabir dam, Iran). Journal of Hydrology 561: 523-531. 
